\title{
Analytical Study on the Influence of Parasitic Elements in a Memristor
}

\author{
Kristopher J. Chandía $\mathbb{D}^{\mathrm{D}},{ }^{1}$ Mauro Bologna $\mathbb{D},{ }^{2}$ and Bernardo Tellini ${ }^{3}$ \\ ${ }^{1}$ Escuela Universitaria de Ingeniería Eléctrica-Electrónica, Universidad de Tarapacá, Arica, Chile \\ ${ }^{2}$ Instituto de Alta Investigación (IAI), Universidad de Tarapacá, Casilla 7-D, Arica, Chile \\ ${ }^{3}$ Dipartimento di Ingegneria dell'Energia, dei Sistemi, del Territorio e delle Costruzioni, University of Pisa, 56122 Pisa, Italy \\ Correspondence should be addressed to Kristopher J. Chandía; kchandia@gmail.com
}

Received 15 November 2017; Revised 31 December 2017; Accepted 7 February 2018; Published 7 March 2018

Academic Editor: Yakov Strelniker

Copyright (C) 2018 Kristopher J. Chandía et al. This is an open access article distributed under the Creative Commons Attribution License, which permits unrestricted use, distribution, and reproduction in any medium, provided the original work is properly cited.

\begin{abstract}
We study a memristive circuit with included parasitic elements, such as capacitance and inductance. In the multiple-scale scheme, we analytically show how the parasitic elements affect the voltage and the current. Finally, we provide an analytical expression for the intersection point coordinates, through which we discuss the functional behavior of the pinched hysteresis loop versus the operating frequency and the parasitic elements.
\end{abstract}

\section{Introduction}

In 1971, Chua postulated the existence of a fourth fundamental element circuit, which was termed memristor [1]. In 2008, Williams and his team of HP laboratories announced the first successful realization of a memristor [2]. Later, many properties and applications have been visualized for memristor, including potential construction of high-density nonvolatile memories [3] and artificial neural networks [4,5]. Several researchers proposed models to explain the memristor dynamics [6-10], fingerprints of memristors, and relevant nonlinear properties. In $[8,9]$, some essential features of memristors (fingerprints) are discussed. Both papers highlight three significant fingerprints: pinched hysteresis loop, the area of hysteresis lobe decreases as frequency increases, and pinched hysteresis loop becomes a single-valued function at infinite frequency. The main purpose of this work is to study the dynamics of a memristor considering their parasitic elements. Through the analytical multiple-scale analysis, we show how parasitic elements affect the fingerprints of memristors and we validate the results with those obtained from a numerical analysis. In particular, we show how the coordinates of the intersection point change as a result of a dominating capacitive or inductive behavior according to the adopted parameter values. The paper is organized as follows. In Section 2, we consider the HP's memristor [2] and we write the equation that governs the memristor circuit when parasitic elements are added, that is, capacitance and inductance. In Section 3, we solve the nonlinear equation driving the circuit using the multiple-scale technique. In Section 4, we find the analytical expression for the displacement of the intersection point of the pinched hysteresis loop and we provide a discussion on the basis of numerical simulations and circuital analysis. Finally, we draw some conclusions.

\section{Memristor with Parasitic Elements}

In the work in [11], the authors considered a realistic memristive circuit, where parasitic elements are included (Figure 1). That work studied the fingerprints of two memristive systems (thermistors), obtaining results through experimental measurements and numerical simulations. The usual fingerprints for ideal memristive systems are modified by the existence of parasitic elements such as inductance $L$, capacitance $C$, small DC current source $I_{p}$, and DC voltage source $V_{p}$ as shown in Figure 1. We consider a simplified version of the previous circuit as shown in Figure 2, where $V_{p}$ and $I_{p}$ are neglected. 


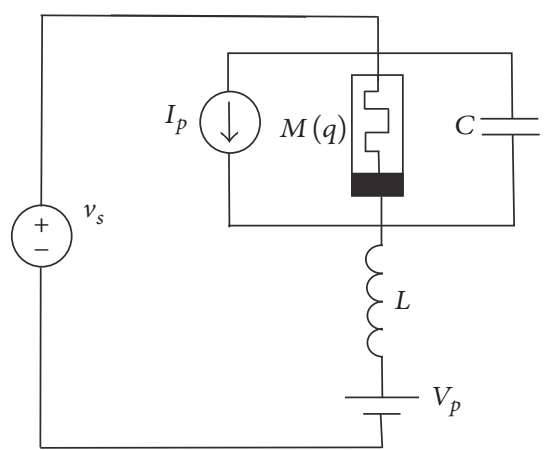

FIgURE 1: A memristor considering its parasitic elements: inductance $L$, capacitance $C$, small DC current source $I_{p}$, and small DC voltage source $V_{p}$.

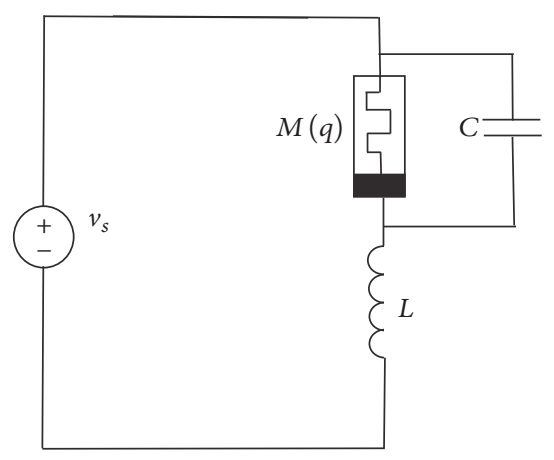

Figure 2: A memristor considering its parasitic inductance and capacitance.

Before building the equation for the circuit of Figure 2, we recall that the memristance is defined according to the literature as [1]

$$
v_{M}=\frac{d}{d t} \phi_{M}(q)=\frac{d}{d q} \phi_{M}(q) \frac{d q}{d t}=M(q) \frac{d q}{d t},
$$

where $M(q)$ is the memristance by definition. According to Kirchhoff's current law [12], the following expression must be satisfied:

$$
\frac{\mathrm{d} q_{L}}{d t}=\frac{d q}{d t}+\frac{d q_{\mathrm{C}}}{d t}
$$

where $q_{C}$ represents electric charge in the capacitor. Due to the fact that the capacitor is connected in parallel with the ideal memristor, we are able to establish a relationship between $q_{C}$ and $\phi_{M}(q)$; that is,

$$
\frac{q_{C}}{C}=\frac{d \phi_{M}}{d t} .
$$

Using the previous result, we may rewrite (2) as

$$
\frac{d q_{L}}{d t}=\frac{d q}{d t}+C \frac{d^{2} \phi_{M}}{d t^{2}}
$$

We are now in a position to write the driving equation of the circuit of Figure 2. Indeed, from Kirchhoff's voltage law [12], we have

$$
\begin{aligned}
v_{s}(t) & =v_{L}(t)+v_{M}(t)=L \frac{d^{2} q_{L}}{d t^{2}}+\frac{d \phi_{M}}{d t} \\
& =L \frac{d^{2} q_{L}}{d t^{2}}+M(q) \frac{d q}{d t} .
\end{aligned}
$$

Inserting (4) into (5) and with the help of (1), we obtain the circuit equation

$$
\begin{aligned}
v_{s}(t)= & L C M(q) \frac{d^{3} q}{d t^{3}}+L \frac{d^{2} q}{d t^{2}}\left(1+3 C \frac{d M(q)}{d q} \frac{d q}{d t}\right) \\
& +M(q) \frac{d q}{d t}+L C \frac{d^{2} M(q)}{d q^{2}}\left(\frac{d q}{d t}\right)^{3},
\end{aligned}
$$

where $L$ and $C$ are the parasitic inductance and capacitance, respectively, and $M(q)$ represents the memristance. We may find the circuit equation in terms of the function $\phi_{M}$ integrating (5) with respect to the time. We obtain

$$
\int v_{s}(t) d t=\phi_{M}(q)+L \frac{d q_{L}}{d t}
$$

Using (4), (7) can be rewritten as follows:

$$
\int v_{s}(t) d t=L \frac{d q}{d t}+\frac{1}{\omega_{0}^{2}} \frac{d^{2} \phi_{M}}{d t^{2}}+\phi_{M}, \quad \omega_{0} \equiv \frac{1}{\sqrt{L C}} .
$$

We now focus on HP's memristor. The analytical expression of memristance is given by the following expression:

$$
M(q)=R_{\mathrm{off}}\left(1-\frac{\mu_{v} R_{\mathrm{on}}}{D^{2}} q(t)\right) .
$$

Here $D$ depends on the memristor length, whereas $\mu_{\nu}$ denotes the ion mobility, $R_{\mathrm{on}}$ denotes the resistance for completely doped memristor, and $R_{\text {off }}$ denotes the resistance for completely undoped memristor. We can determine an expression for $\phi_{M}$, given by

$$
\phi_{M}=\int M(q) d q=R_{\mathrm{off}}\left(q(t)-\frac{q^{2}(t)}{2 q_{0}}\right),
$$

where $q_{0}=D^{2}\left(\mu_{v} R_{\text {on }}\right)^{-1}$ and the integration constants are set to zero. Next, we consider a periodic voltage source,

$$
v_{s}(t)=V_{0} \cos (\omega t),
$$


and define the dimensionless quantities via the parameter $\phi_{0}=R_{\text {off }} q_{0}$ :

$$
\begin{aligned}
f & =\frac{V_{0}}{\omega \phi_{0}}, \\
r & =\frac{1}{C R_{\text {off }} \omega}, \\
\tau & =\omega t, \\
Q & =\frac{q}{q_{0}}, \\
\Phi & =\frac{\phi}{\phi_{0}} .
\end{aligned}
$$

In terms of the dimensionless quantities, (9) and (10) can be rewritten as

$$
\begin{aligned}
& M(Q)=\frac{\phi_{0}}{q_{0}}[1-Q(t)] \\
& \Phi(Q)=\left[Q(t)-\frac{Q^{2}(t)}{2}\right] .
\end{aligned}
$$

We can invert (14) and, for $Q$, we obtain

$$
Q=1 \pm \sqrt{1-2 \Phi}
$$

Imposing the condition that $Q$ takes real values, it is necessary that $\Phi<1 / 2$. Plugging (15) into (8) and making the time transformation $t=\tau / \omega$, we obtain the equation for the function $\Phi$ :

$$
\varepsilon \frac{d^{2} \Phi}{d \tau^{2}} \mp \frac{\varepsilon r}{\sqrt{1-2 \Phi}} \frac{d \Phi}{d \tau}+\Phi=f \sin \tau
$$

where the parameter $\varepsilon$ is defined as

$$
\varepsilon=\frac{\omega^{2}}{\omega_{0}^{2}}
$$

To determine the sign in (16) on which we will focus in the next section, we impose the constraint that the memristor has to be a passive memristor. A memristor is passive when the memristance is positive; that is, $M(q)>0$. This condition is satisfied when $q(t)<q_{0}$ or, equivalently, in dimensionless variable, $Q<1$ (see (13)). The condition $Q<1$ implies that we have to choose the negative sign in (15) and consequently the positive sign in (16). This will be the subject of our study in the next section.

\section{Dynamics of a Memristor with Parasitic Elements}

In this section, we study (16) considering the positive sign of the coefficient of the first derivative of $\Phi$, corresponding to the negative sign in (15). We consider $\varepsilon$ as a small parameter due to the fact that parasitic elements have small values of capacitance and inductance (see $[11,13]$ for numerical data).
In other words, we consider the case $\omega_{0} \gg \omega$ or $\varepsilon \ll 1$. For what we said, our starting point is the exact equation

$$
\varepsilon \frac{d^{2} \Phi}{d \tau^{2}}+\frac{\varepsilon r}{\sqrt{1-2 \Phi}} \frac{d \Phi}{d \tau}+\Phi=f \sin \tau
$$

We define a suitable time scale $T=\tau / \sqrt{\varepsilon}$ and a new perturbative parameter $\delta=\sqrt{\varepsilon}$. Replacing $\tau$ by $T \sqrt{\varepsilon}$ and $\sqrt{\varepsilon}$ by $\delta$ in (18), we obtain

$$
\frac{d^{2} \Phi}{d T^{2}}+\frac{\delta r}{\sqrt{1-2 \Phi}} \frac{d \Phi}{d T}+\Phi=f \sin \tau
$$

We stress that (19) contains only one parameter $\delta r$ and two time scales $T$ and $\tau$. We are now in the position to apply the multiple-scale method to (19). Following the procedure given in [14], we postulate a solution of the form

$$
\Phi(T)=\sum_{n=0}^{\infty} \delta^{n} \Phi_{n}\left(T^{+}, \tau\right)
$$

where $T^{+}=T\left(1+a_{2} \delta^{2}+\cdots\right)$ is the fast variable and $\tau=\delta T$ is the slow variable. The first two derivatives of $\Phi(T)$ at the firstorder approximation are given by (the reader can find these results in great detail in chapter IV of [14])

$$
\begin{aligned}
\frac{d \Phi}{d T} & =\frac{\partial \Phi_{0}}{\partial T^{+}}+\delta\left(\frac{\partial \Phi_{1}}{\partial T^{+}}+\frac{\partial \Phi_{0}}{\partial \tau}\right)+O\left(\delta^{2}\right) \\
\frac{d^{2} \Phi}{d T^{2}} & =\frac{\partial^{2} \Phi_{0}}{\partial T^{+2}}+\delta\left(\frac{\partial^{2} \Phi_{1}}{\partial T^{+2}}+2 \frac{\partial^{2} \Phi_{0}}{\partial T^{+} \partial \tau}\right)+O\left(\delta^{2}\right)
\end{aligned}
$$

Using the expansion in (20), we can write

$$
\frac{1}{\sqrt{1-2 \Phi(T)}}=\frac{1}{\sqrt{1-2 \Phi_{0}}}+\frac{\delta \Phi_{1}}{\left(1-2 \Phi_{0}\right)^{3 / 2}}+O\left(\delta^{2}\right) .
$$

To the zeroth-order expansion, we obtain the following differential equation:

$$
\frac{d^{2}}{d T^{+2}} \Phi_{0}\left(T^{+}, \tau\right)+\Phi_{0}\left(T^{+}, \tau\right)=f \sin \tau
$$

The solution for $\Phi_{0}\left(T^{+}, \tau\right)$ is given by

$$
\begin{aligned}
\Phi_{0}\left(T^{+}, \tau\right)= & A(\tau) \cos \left(T^{+}\right)+B(\tau) \sin \left(T^{+}\right) \\
& +\frac{f}{1-\delta^{2}} \sin \tau
\end{aligned}
$$

where $A$ and $B$ are functions depending on the slow variable $\tau$. At the order $\delta$, using (22), we obtain the following differential equation:

$$
\frac{d^{2} \Phi_{1}}{d T^{+2}}+\Phi_{1}=-\frac{r}{\sqrt{1-2 \Phi_{0}}} \frac{\partial \Phi_{0}}{\partial T^{+}}-2 \frac{\partial^{2} \Phi_{0}}{\partial T^{+} \partial \tau} .
$$

In order to ensure the passivity of memristance $M(q)$, we know that $Q<1$ and consequently $\Phi_{0}<1 / 2$. We now assume 
that $\Phi_{0}$ is sufficiently far from the value $1 / 2$ in such a way that we may expand square root as

$$
\frac{1}{\sqrt{1-2 \Phi_{0}}} \simeq\left(1+\Phi_{0}\right)
$$

With this further approximation, evaluating (26), we end up with

$$
\begin{aligned}
\frac{d^{2} \Phi_{1}}{d T^{+2}}+\Phi_{1}= & -\left[2 \frac{d B(\tau)}{d \tau}+r B(\tau)\right] \cos \left(T^{+}\right) \\
& +\left[2 \frac{d A(\tau)}{d \tau}+r A(\tau)\right] \sin \left(T^{+}\right) \\
& +\frac{r}{2} \sin \left(2 T^{+}\right)\left[A(\tau)^{2}-B(\tau)^{2}\right] \\
& -r A(\tau) B(\tau)
\end{aligned}
$$

To avoid secular terms, we must vanish the resonant term coefficients, that is, the coefficients of $\cos T^{+}$and $\sin T^{+}$ in (28). This procedure leads to the following system of differential equations:

$$
\begin{aligned}
& \frac{d A(\tau)}{d \tau}+\frac{r}{2} A(\tau)=0, \\
& \frac{d B(\tau)}{d \tau}+\frac{r}{2} B(\tau)=0 .
\end{aligned}
$$

The solutions for $A(\tau)$ and $B(\tau)$ are given by

$$
\begin{aligned}
& A(\tau)=C_{1} \exp \left[-\frac{r}{2} \tau\right], \\
& B(\tau)=C_{2} \exp \left[-\frac{r}{2} \tau\right],
\end{aligned}
$$

where $C_{1}$ and $C_{2}$ are arbitrary constants depending on initial conditions for $\Phi_{0}$. By evaluating the initial conditions, we obtain

$$
\begin{aligned}
& C_{1}=\Phi_{\mathrm{ini}}, \\
& C_{2}=\left(\dot{\Phi}_{\mathrm{ini}}-\frac{f \delta}{\left(1-\delta^{2}\right)}+\frac{r \delta}{2} \Phi_{\mathrm{ini}}\right),
\end{aligned}
$$

where $\Phi_{0}(T=0)=\Phi_{\text {ini }}$ and $\left(d \Phi_{0} / d T\right)(T=0)=\dot{\Phi}_{\text {ini }}$. Finally, the expression for $\Phi_{0}(T)$ is given by

$$
\begin{aligned}
& \Phi_{0}(T, \tau)=\exp \left[-\frac{r}{2} \tau\right]\left[\Phi_{\mathrm{ini}} \cos T^{+}\right. \\
& \left.+\left(\frac{r \delta}{2} \Phi_{\mathrm{ini}}+\dot{\Phi}_{\mathrm{ini}}-\frac{f \delta}{\left(1-\delta^{2}\right)}\right) \sin T^{+}\right]+\frac{f}{1-\delta^{2}} \\
& \cdot \sin \tau,
\end{aligned}
$$

where $\tau=\delta T$. In Figure 3, we compare the analytical expression obtained in (32) with a numerical simulation. The figure shows an excellent agreement between numerical and analytical solutions even during the transient period.

Using (15), we plot the electric charge and compare it with the numerical solution. Figures 4 and 5 show a good agreement between numerical and analytical solutions. In Figure 4, we consider a shorter interval of time to appreciate the wavy part that is reproduced by the analytical solution.

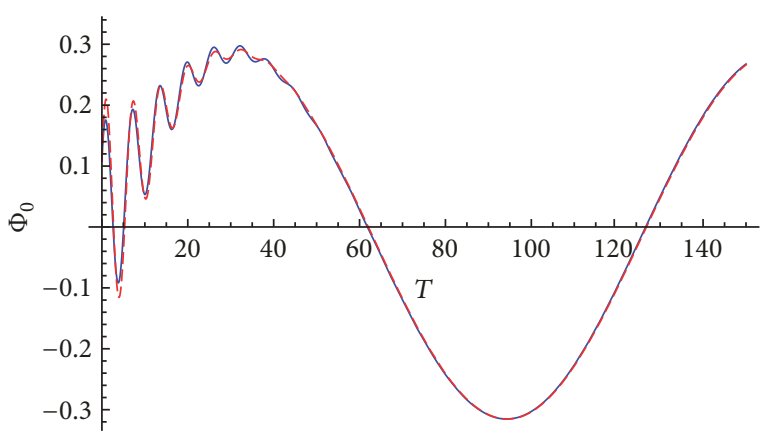

Figure 3: First term $\Phi_{0}$ versus time $T$ obtained in (32) (solid line) and numerical solution (dashed line). The parameter values are $\Phi_{\text {ini }}=0.15, \dot{\Phi}_{\text {ini }}=0.1, \delta=0.05, f=0.3$, and $r=3.5$. The axis units are dimensionless.

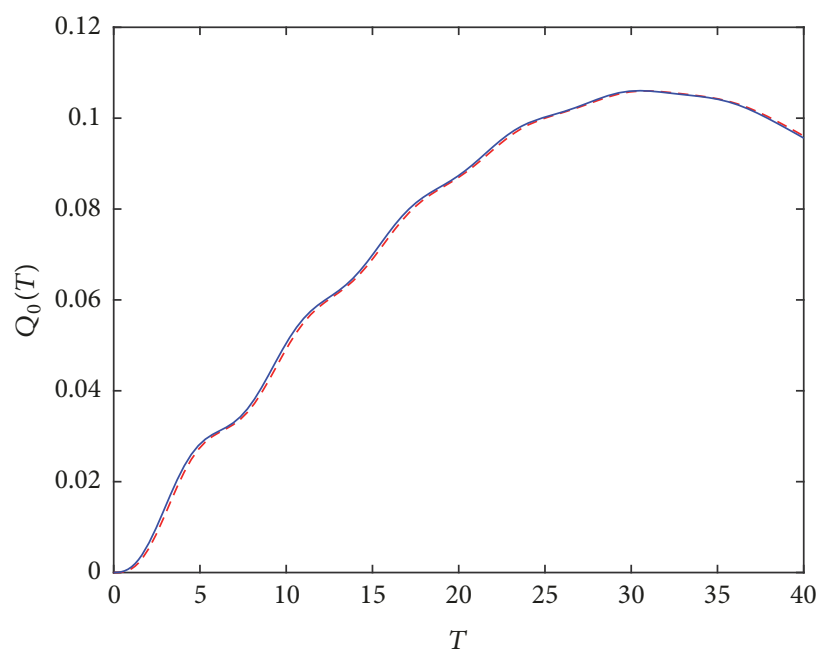

Figure 4: First term $Q_{0}$ versus time $T$ using (15) and numerical solution (dashed line) in the transient regime. The parameter values are $Q_{\text {ini }}=0, \dot{Q}_{\text {ini }}=0, \delta=0.05, f=0.1$, and $r=3.5$. The axis units are dimensionless.

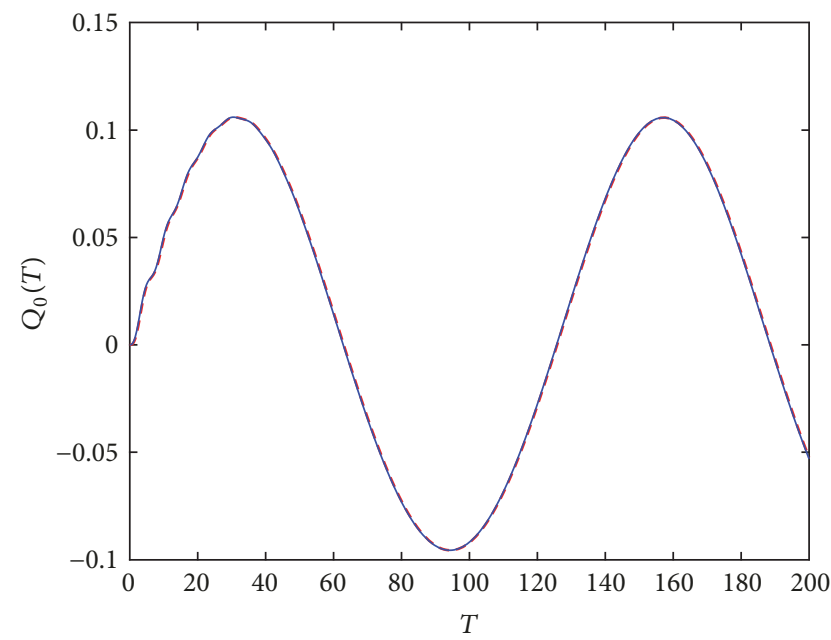

FIgURE 5: First term $Q_{0}$ versus time $T$ using (15) and numerical solution (dashed line). The parameter values are $Q_{\mathrm{ini}}=0, \dot{Q}_{\mathrm{ini}}=0$, $\delta=0.05, f=0.1$, and $r=3.5$. The axis units are dimensionless. 


\section{Behavior of Pinched Hysteresis Loop}

As discussed in [11], parasitic elements modify the pinched hysteresis loop with respect to the loop of an ideal memristor. In this section, we will find the analytical coordinates of the intersection point. Under steady-state condition, we are allowed to neglect the exponential terms in (32). We consider as memristor the elements described in Figure 2, namely, the ideal memristor and the parasitic elements. Rewriting the electrical current (see (4)) and voltage (see (11)) of the memristor in terms of the dimensionless quantities given by (12), we have

$$
\begin{aligned}
& \frac{d Q_{L}}{d \tau} \\
& \quad=\frac{f}{1-\delta^{2}}\left[\frac{\cos (\tau)}{\sqrt{1-\left(2 f /\left(1-\delta^{2}\right)\right) \sin (\tau)}}-\frac{\sin (\tau)}{r}\right], \\
& V_{s}(\tau)=f \cos (\tau),
\end{aligned}
$$

where $d Q_{L} / d \tau$ is the electrical current and $V_{s}(\tau)$ is the voltage of the HP memristor in dimensionless notation. To determine the intersection point located in the $i-v$ curve, we need to find the times $\tau_{1}$ and $\tau_{2}$ such that

$$
\begin{aligned}
\left.\frac{d Q_{L}}{d \tau}\right|_{\tau=\tau_{1}} & =\left.\frac{d Q_{L}}{d \tau}\right|_{\tau=\tau_{2}}, \\
V_{s}\left(\tau_{1}\right) & =V_{s}\left(\tau_{2}\right) .
\end{aligned}
$$

For the sake of brevity, we will focus on the first quadrant, where both cosines and sinus are positive quantities. From condition (35), we obtain

$$
\tau_{1}=2 n \pi \pm \tau_{2}
$$

The relationship $\tau_{1}=2 n \pi+\tau_{2}$ gives the identical solution corresponding to the fact that electrical current and voltage are periodic functions in stationary regime. Substituting the second equality, $\tau_{1}=2 n \pi-\tau_{2}$, into the electrical current expression (34), we obtain the following equation:

$$
\begin{array}{r}
\frac{\sqrt{1-x^{2}}}{\sqrt{1-a x}}-\frac{\sqrt{1-x^{2}}}{\sqrt{1+a x}}=\frac{2 x}{r}, \\
x \equiv \sin \left(\tau_{2}\right), a \equiv \frac{2 f}{1-\delta^{2}} .
\end{array}
$$

We may further assume that $a \ll 1$, and thus we can write $\sqrt{1-a x} \sim(1-a x / 2)$, so

$$
x=\sin \left(\tau_{2}\right)=\sqrt{1-\frac{4}{a^{2} r^{2}}} .
$$

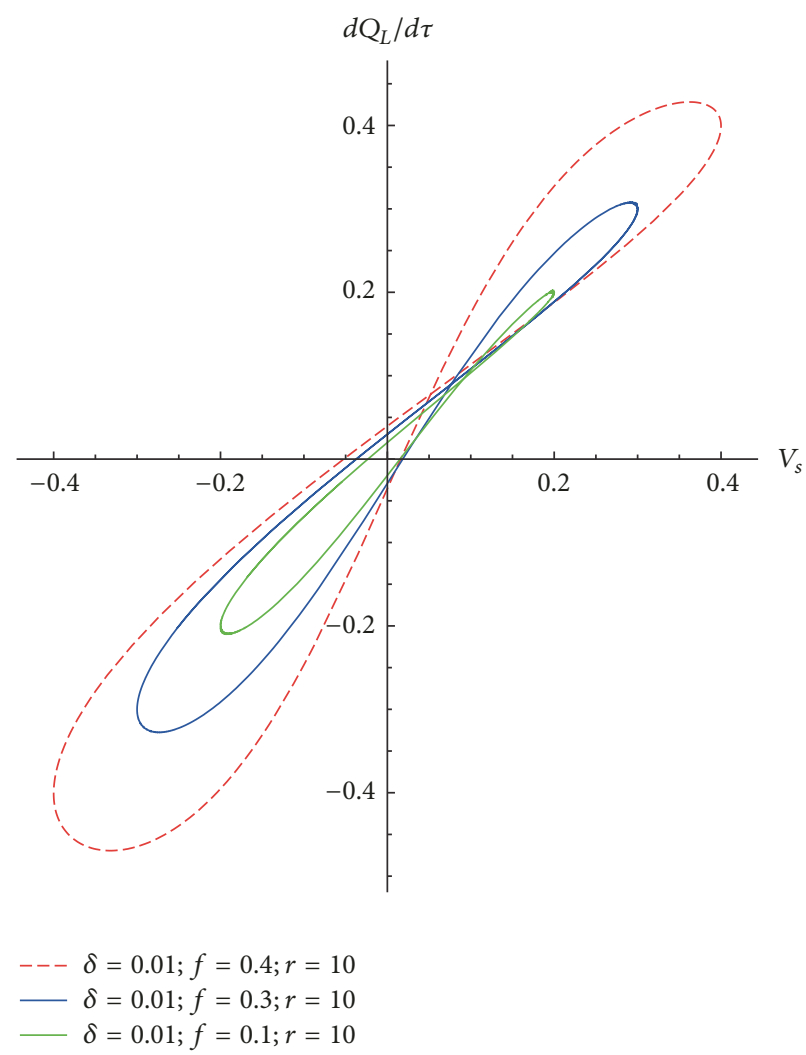

Figure 6: Plot of the pinched hysteresis loop as function of the source voltage amplitude $f$.

In the range of validity of (38), the intersection point coordinates are

$$
\begin{aligned}
& \left.\frac{d Q_{L}}{d \tau}\right|_{\tau=\tau_{2}} \\
& =\frac{f}{1-\delta^{2}}\left[\frac{2}{a r \sqrt{1-\left(2 f /\left(1-\delta^{2}\right)\right) \sqrt{1-4 / a^{2} r^{2}}}}\right. \\
& \left.-\frac{\sqrt{1-4 / a^{2} r^{2}}}{r}\right], \\
& V_{s}\left(\tau_{2}\right)=\frac{2 f}{a r} .
\end{aligned}
$$

Note that, taking the limit $L, C \rightarrow 0, r \rightarrow \infty$ and $\delta \rightarrow 0$ (see (12) and (17)) and from (39) and (40) we recover the ideal memristor with the intersection point located at the origin. In Figure 6, we plot several pinched hysteresis loops for different amplitude of the voltage source. The coordinates of the intersection point are well described by (38) and (39). For small $\delta$ and $f$, we compare our analytical solution with the numerical result as shown in Figure 7. Analogously, we may proceed with the third quadrant. In Figure 7, we can appreciate two lobes; the direction of the arrows represents 


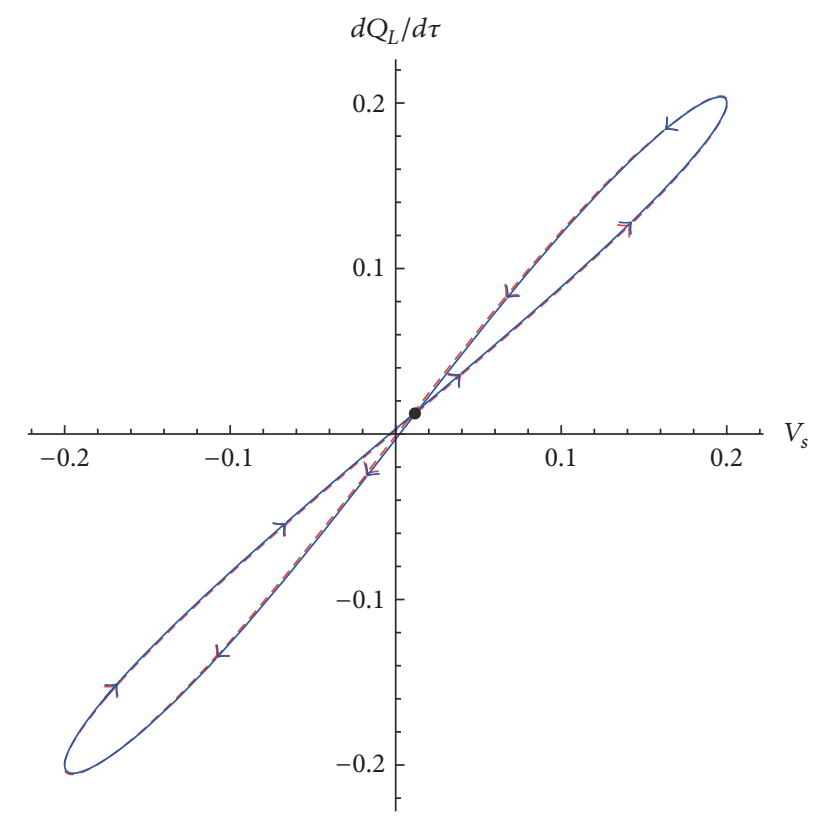

Figure 7: Plot of $i-v$ curve for $\delta=0.01, f=0.2$, and $r=65$ in the steady-state condition. Numerical results (dashed line) and analytical solution (solid line). The axis units are dimensionless.

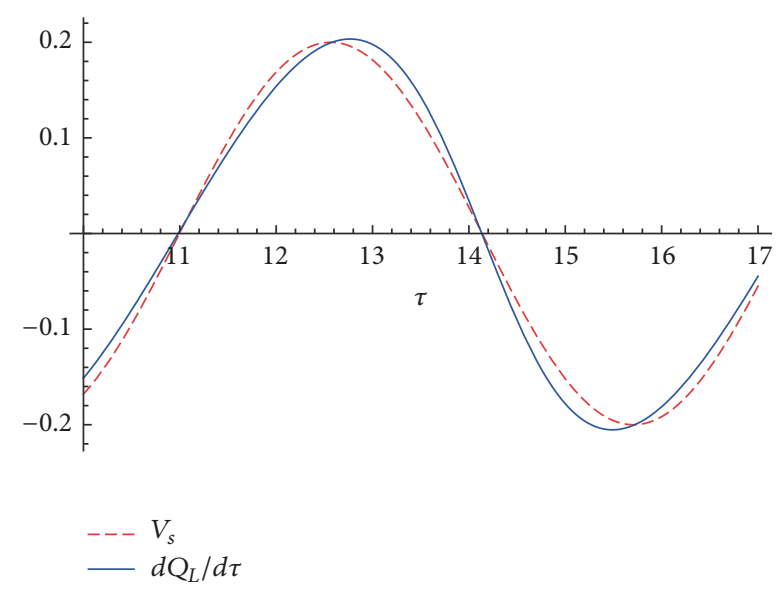

FIgURE 8: Plot of the voltage (dashed line) and the electrical current for a period in stationary regime. $\delta=0.01, f=0.2$, and $r=65$.

the change of the variables voltage and current. In the upper lobe, the direction of the arrows (counterclockwise) is similar to the case found in an ohmic-inductive circuit. Conversely, in the lower lobe, the direction of the arrows is clockwise, similar to the case found in an ohmic-capacitive circuit. For the values used in Figure 7, the capacitive lobe (lower) is greater than the inductive lobe, so the current leads the voltage most of the time in a period for the stationary regime. To clarify this point, we plot the voltage and electrical current versus time to show this fact.

In Figure 8, we can appreciate how the current alternately leads and lags the voltage during a period. This fact is due to the nonlinear nature of memristor. Indeed the electrical current is not a pure sinusoidal signal and so we can find some

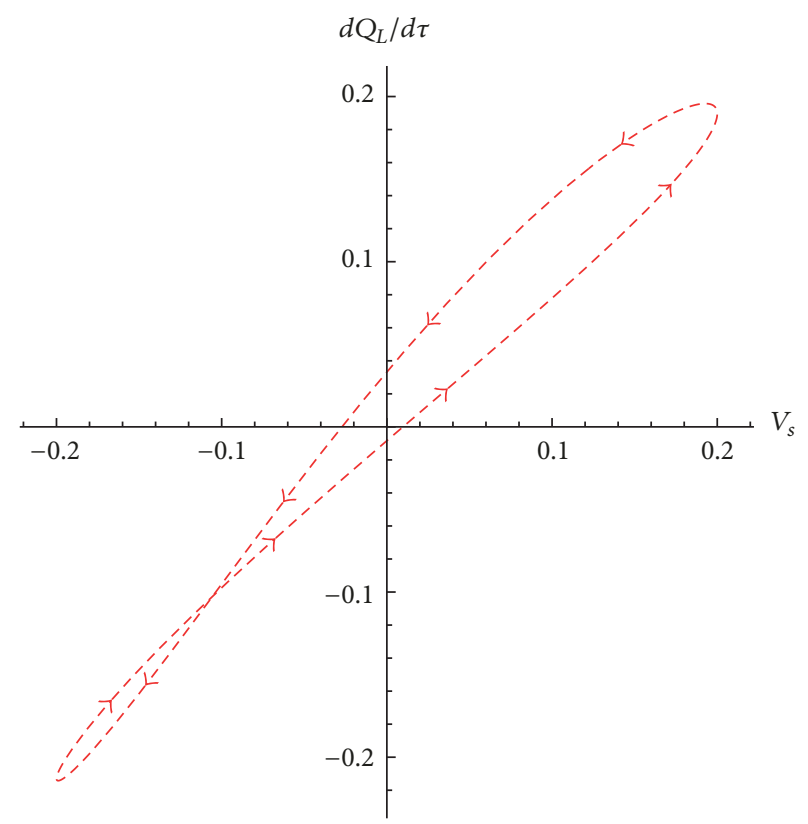

Figure 9: Plot of $i-v$ curve for $\delta=0.1, f=0.2$, and $r=15$ in the steady-state condition (only numerical results are shown). The axis units are dimensionless.

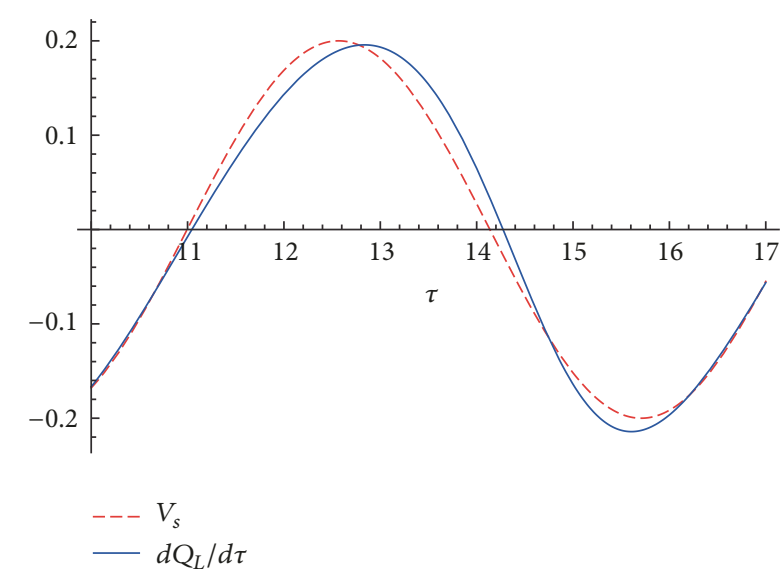

FIGURE 10: Plot of the voltage (dashed line) and the electrical current for a period in stationary regime. $\delta=0.1, f=0.2$, and $r=15$.

time intervals, where the voltage lags the current and in other cases the voltage leads the current.

In Figure 9, we show the numerical results for $\delta=0.1$, $f=0.2$, and $r=15$. We can see that the intersection point is located in the third quadrant according to a dominating inductive behavior as also shown in Figure 10.

\section{Conclusion}

In this paper, we considered a model of a memristor with included parasitic elements. We analyzed a system composed of a parasitic inductance in series with the parallel between an ideal memristance and a parasitic capacitance. Using the multiple-time-scale approach, we studied the dynamics 
of the flux and electric charge of memristor. The parasitic elements considered in the model modify the pinched hysteresis loop. We discussed the functional behavior of the pinched hysteresis loop versus the operating frequency and the parasitic elements. In particular, we showed that the intersection point moves apart from the origin of the $i$ - $v$ plane and we analytically determined its coordinates. Finally, our theoretical analysis is shown to be in excellent agreement with the numerical simulations.

\section{Conflicts of Interest}

The authors declare that they have no conflicts of interest.

\section{Acknowledgments}

Kristopher J. Chandía and Mauro Bologna acknowledge financial support from UTA Mayor (Project no. 8731-15).

\section{References}

[1] L. O. Chua, "Memristor-the missing circuit element," IEEE Transactions on Circuit Theory, vol. 18, no. 5, pp. 507-519, 1971.

[2] D. B. Strukov, G. S. Snider, D. R. Stewart, and R. S. Williams, "The missing memristor found," Nature, vol. 453, pp. 80-83, 2008.

[3] M.-F. Chang, A. Lee, P.-C. Chen et al., "Challenges and circuit techniques for energy-efficient on-chip nonvolatile memory using memristive devices," IEEE Journal on Emerging and Selected Topics in Circuits and Systems, vol. 5, no. 2, pp. 183-193, 2015.

[4] M. Hu, Y. Chen, J. J. Yang, Y. Wang, and H. Li, "A Compact Memristor-Based Dynamic Synapse for Spiking Neural Networks," IEEE Transactions on Computer-Aided Design of Integrated Circuits and Systems, vol. 36, no. 8, pp. 1353-1366, 2017.

[5] L. Wang, M. Duan, and S. Duan, "Memristive perceptron for combinational logic classification," Mathematical Problems in Engineering, vol. 2013, Article ID 625790, 2013.

[6] R. Zhu, S. Chang, H. Wang, Q. Huang, J. He, and F. Yi, "A Versatile and Accurate Compact Model of Memristor with Equivalent Resistor Topology," IEEE Electron Device Letters, 2017.

[7] A. Ascoli, V. Ntinas, R. Tetzlaff, and G. C. Sirakoulis, "Closedform analytical solution for on-switching dynamics in a $\mathrm{TaO}$ memristor," IEEE Electronics Letters, vol. 53, no. 16, pp. 11251126, 2017.

[8] S. P. Adhikari, M. P. Sah, H. Kim, and L. O. Chua, "Three fingerprints of memristor," IEEE Transactions on Circuits and Systems II: Express Briefs, vol. 60, no. 11, pp. 3008-3021, 2013.

[9] D. Biolek, Z. Biolek, V. Biolkova, and Z. Kolka, "Some fingerprints of ideal memristors," in Proceedings of the 2013 IEEE International Symposium on Circuits and Systems, ISCAS 2013, pp. 201-204, Beijing, China, May 2013.

[10] K. J. Chandia, M. Bologna, and B. Tellini, "Multiple Scale Approach to Dynamics of an LC Circuit With a ChargeControlled Memristor," IEEE Transactions on Circuits and Systems II: Express Briefs, vol. 65, no. 1, pp. 120-124, 2018.
[11] M. P. Sah, C. Yang, H. Kim, B. Muthuswamy, J. Jevtic, and L. Chua, "A generic model of memristors with parasitic components," IEEE Transactions on Circuits and Systems I: Regular Papers, vol. 62, no. 3, pp. 891-898, 2015.

[12] C. Desoer and E. S. Kuh, Basic Circuit Theory, MacGraw-Hill, 1969.

[13] C. R. Paul, "Introduction to Electromagnetic Compatibility: Second Edition," Introduction to Electromagnetic Compatibility: Second Edition, pp. 1-983, 2006.

[14] J. Kevorkian and J. D. Cole, Multiple Scale and Singular Perturbation Methods, Springer, Berlin, Germany, 1996. 


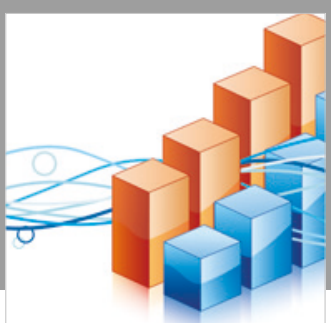

Advances in

Operations Research

\section{-n-m}
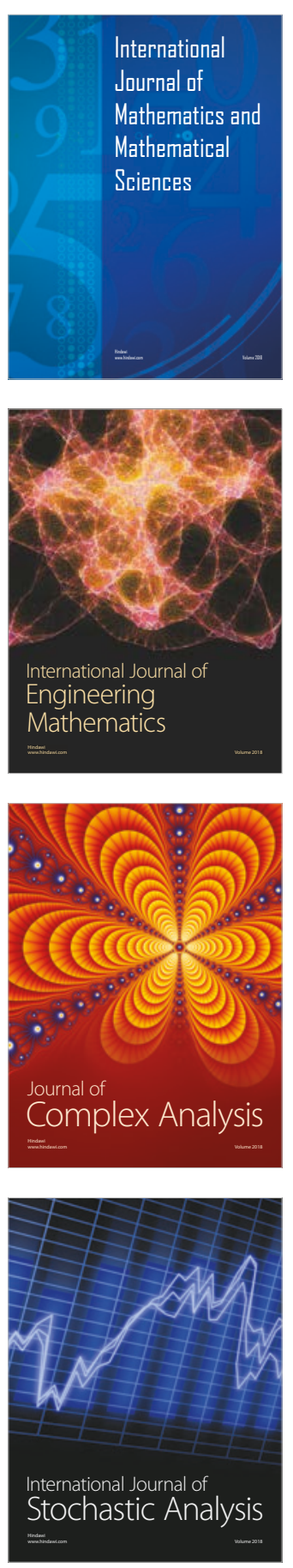
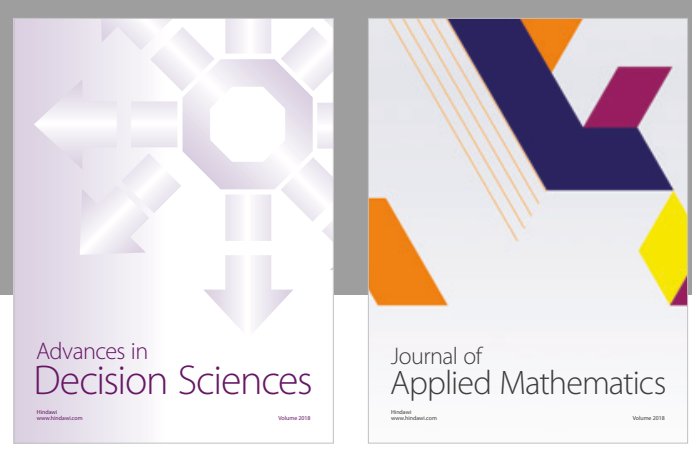

Journal of

Applied Mathematics
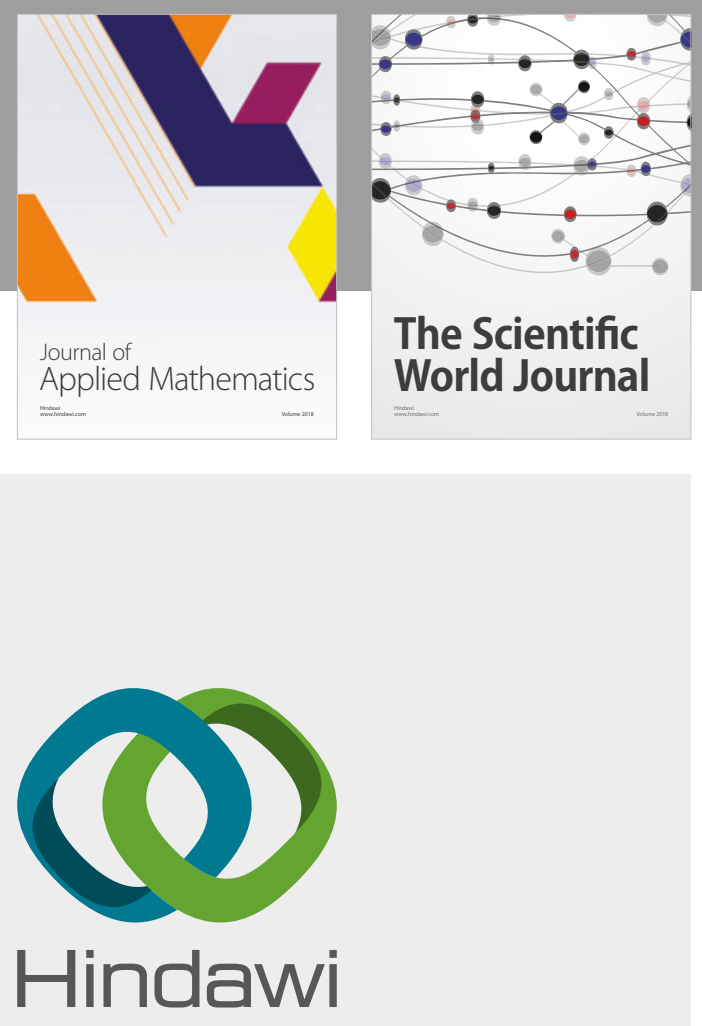

Submit your manuscripts at

www.hindawi.com

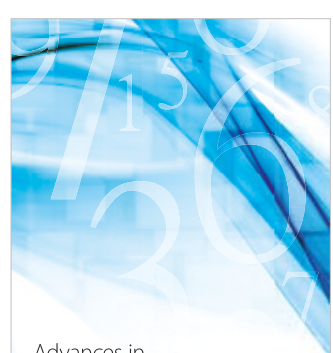

Advances in
Numerical Analysis
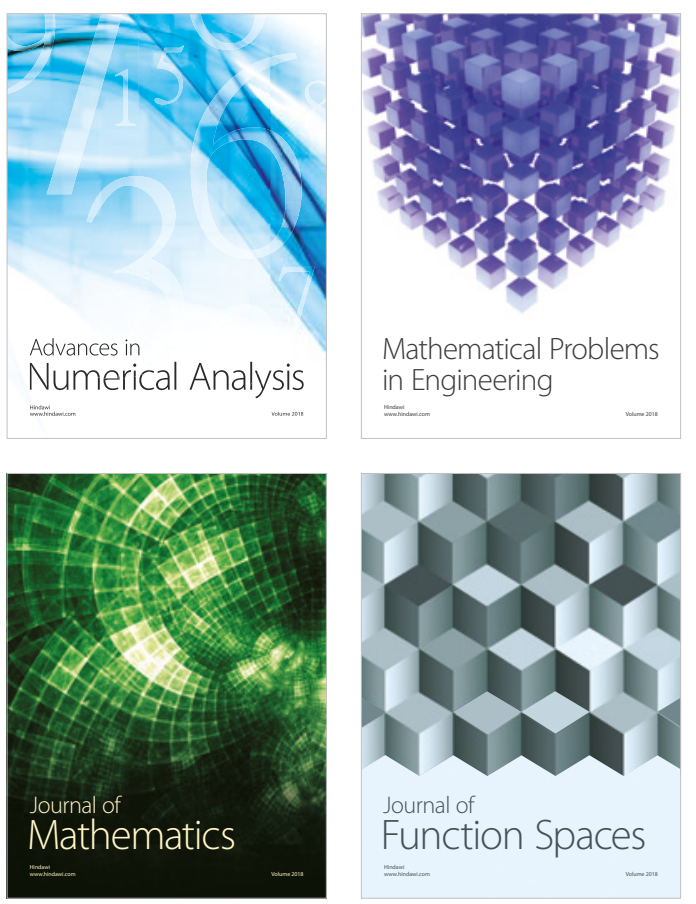

Mathematical Problems in Engineering

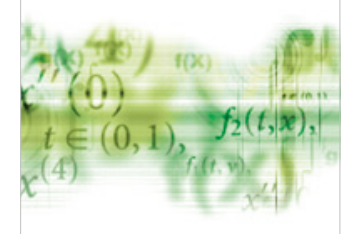

International Journal of

Differential Equations

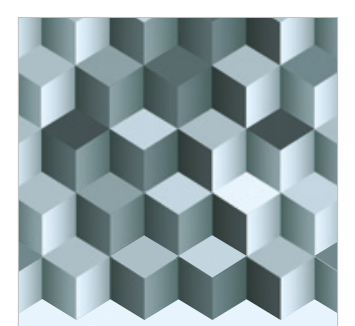

Journal of

Function Spaces

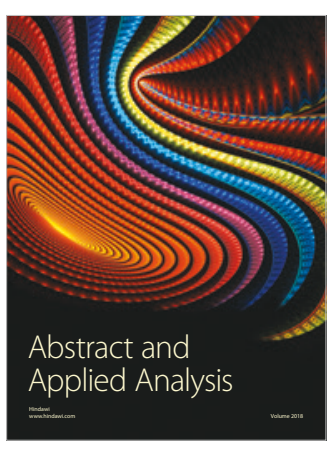

The Scientific

World Journal

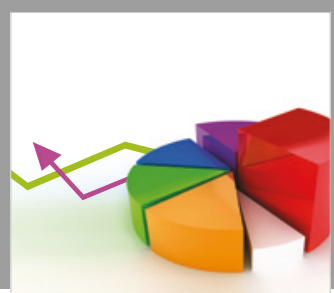

Journal of

Probability and Statistics
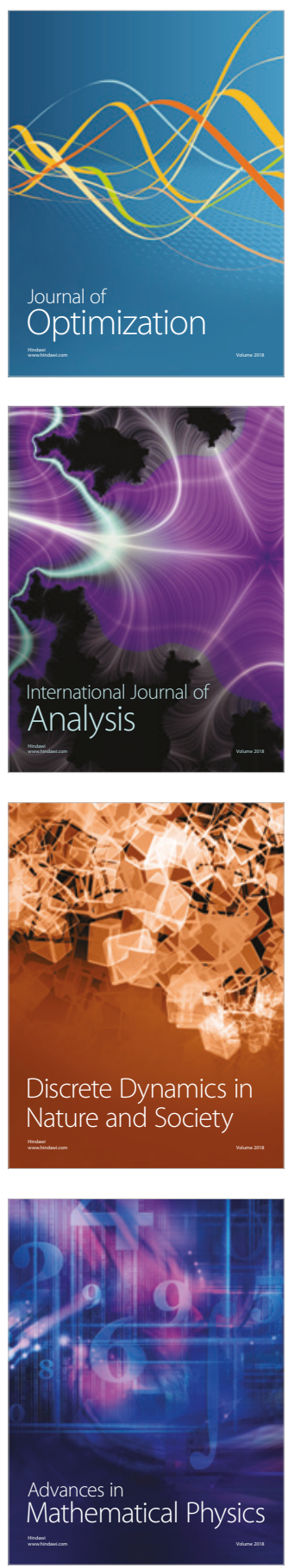